\title{
Health expenditure comparison of extended-release metoprolol succinate and immediate-release metoprolol tartarate
}

This article was published in the following Dove Press journal:

ClinicoEconomics and Outcomes Research

4 February 2012

Number of times this article has been viewed

\author{
Varun Vaidya \\ Pranav Patel \\ College of Pharmacy and \\ Pharmaceutical Sciences, University of \\ Toledo, Toledo, OH, USA
}

Background: Metoprolol, a selective beta-1 blocker, is available in two different salt forms in the market - metoprolol succinate (MS) and metoprolol tartarate (MT). Both the formulations are Food and Drug Administration approved for the treatment of hypertension. Several studies have shown similar efficacies between the two salts; however, they differ in their pharmacokinetic properties and are therefore priced differently. The primary objective of this study was to compare the overall health care expenditures of hypertensive patients on MT and MS to see if the price difference in the two preparations is offset by savings in overall expenditure.

Methods: Two cohorts of patients using MT and MS were selected from the 2008 Medical Expenditure Panel Survey. Propensity score matching technique was used to balance the cohorts on various parameters such as demographic information, insurance status, and comorbidity score. Patients using MT were matched to patients using MS on the logit of propensity score using calipers of width equal to 0.2 of the standard deviation of the logit of the propensity score. Multiple regression analysis was carried out to examine the association between health expenditure and type of metoprolol salt, adjusting for other covariates.

Results: A total of 742 patients were found to use metoprolol (MT-388, MS-354). After propensity score matching, a total of 582 patients were left in the sample for final analysis (291 patients in each cohort). The average annual health care expenditure was slightly higher in the MT cohort; however, after adjusting for covariates in a multivariate analysis, the difference was found to be statistically insignificant $(P=0.23)$.

Conclusion: Both the products of metoprolol were found to have similar average annual total health care expenditure; however, MS once a day has higher out-of-pocket cost.

Keywords: hypertension, cost, propensity score

\section{Introduction}

Hypertension is a condition associated with high morbidity and mortality. ${ }^{1}$ It is also a risk factor, albeit, a manageable one, for conditions like stroke, kidney failure, heart failure, and myocardial infarction. ${ }^{2,3}$ As of 2004, one out of every three individuals in the US was diagnosed with hypertension. This translates to 72 million Americans in $2004 .{ }^{4}$ By 2030, an additional 27 million individuals are expected to be diagnosed with hypertension. ${ }^{5}$

The comorbidities, high prevalence rates, and the chronic nature of hypertension generate substantial economic burden for both the patient and the US health care system. The total hypertension-related costs are projected to rise from US\$93.5 million in 2010 to US\$240.1 million in $2030 .^{5}$ Previous research has shown that systematic control of blood pressure can result in considerable cost savings. ${ }^{6,7}$ Additionally, 
pharmacoeconomic analysis and comparative effectiveness research can help in determining cost-effective treatment choices leading to additional cost savings.

The treatment regimen for hypertension is burdensome for the patient in terms of cost and adherence considering the fact that it is a chronic condition that is usually treated with two or more medications. ${ }^{8}$ Research has shown that adherence to a treatment regimen can lead to better clinical outcomes. ${ }^{6,9,10}$ However, in the general population, adherence to all antihypertensive drug classes has been suboptimal, and the adherence to beta-blockers and diuretics has been the lowest amongst all antihypertensive drug classes. ${ }^{6,9}$

To increase adherence to the antihypertensive treatment regimen by reducing the pill burden, many medications are currently available in a once-daily dosage form. However, most of these drugs come at an additional daily cost. Once-daily medications reduce the pill burden and, as a result, may improve adherence by providing more convenience to the patient. ${ }^{6}$ As the clinical outcomes for both the preparations are similar because of the identical active ingredient, it would be interesting to find whether the improvement in adherence can offset the additional cost by producing savings in overall health care expenditure. In this study, the authors attempted to answer this question by analyzing the health care expenditures and the drug expenditures of the two products of metoprolol in the treatment of hypertension.

Metoprolol is a selective beta-1 blocker lacking intrinsic sympathomimetic activity. It is available in two different formulations, each a different salt - metoprolol tartarate (MT), which is taken twice a day, and metoprolol succinate (MS), an extended-release form, taken once a day in the treatment of hypertension. Both MT and MS are Food and Drug Administration (FDA) approved for the treatment of hypertension and angina pectoris; however, MS is also FDA approved for the reduction of mortality in patients with heart failure (New York Heart Association Class II or Class III). ${ }^{11}$ Studies have shown similarity in outcomes between the two products in the treatment of hypertension. ${ }^{12,13}$ However, they are different in terms of their cost. MS has an average wholesale price (AWP) of US $\$ 52.45 / 30$ pills (30-day supply), and MT has an AWP of US $\$ 34.09 / 60$ pills (30-day supply). ${ }^{14}$ Moreover, the acquisition price of both MT and MS could be much lower since both of these drugs are available as generic products.

The main objective of this study was to compare the impact of the metoprolol products on overall health care expenditure and prescription drug expenditure using population-based data of matched cohorts. The authors examined whether a specific type of metoprolol product was associated with changes in total health care expenditures and prescription drug expenditure.

\section{Methods}

Data from the 2008 Medical Expenditure Panel Survey (MEPS) was used to perform a retrospective cross-sectional analysis. MEPS is a nationally representative database maintained by the Agency for Healthcare Research and Quality under the purview of the United States Department of Health and Human Services. It is a database of the results of the large-scale surveys of families and/or individuals, health care providers, employers, and payers.

The medical conditions file HC-120 and the prescribed medicines file HC-118 were used to identify patients being treated for hypertension (International Classification of Diseases, Ninth Revision, Clinical Modification [ICD-9-CM] codes 401-405) using MT or MS. Patients using MT or MS were filtered using the medication name: metoprolol, metoprolol tart*, and metoprolol succ*. The fields that did not have a medication name were then identified using the eleven-digit National Drug Code (NDC) in an effort to not leave out any of the hypertensive patients on MT or MS.

MT and MS are approved for the treatment of hypertension and angina. Since this study was limited to the utility of MT and MS in the treatment of hypertension, patients on MT or MS who had a record of angina (identified by ICD-9 code 413) in addition to a record of hypertension were excluded from the study.

The patient cohorts obtained were then merged with the full-year consolidated data files (HC-121) to get demographic information, as well as the expenditure information of the patients.

\section{Statistical analysis}

After the patients meeting all requirements described in the inclusion/exclusion criteria (Figure 1) were selected, they were divided into two separate groups depending upon the type of metoprolol product (MT or MS). Baseline characteristics were compared between the patient cohorts, and descriptive statistics were calculated. To achieve the balance in baseline characteristics and to account for the selection bias, propensity score matching was applied. Propensity score matching is a technique that aims at adjusting for selection bias in nonexperimental, nonrandomized, and retrospective observational studies like the present one. Propensity score matching allowed mirroring each patient in the MS cohort with a patient with similar predefined characteristics in the 


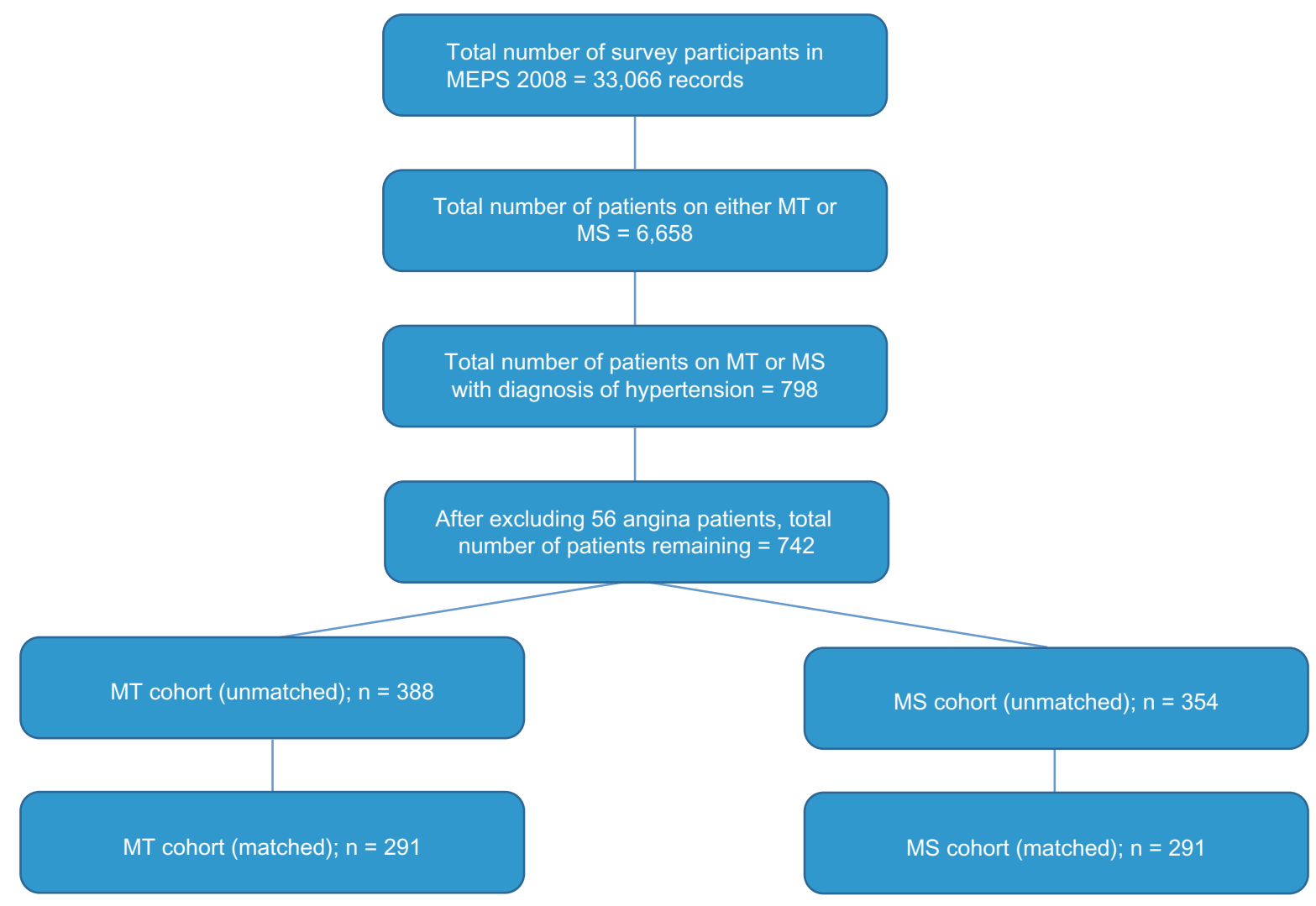

Figure I Patient selection criteria of MT and MS cohorts.

Abbreviations: MEPS, Medical Expenditure Panel Survey; MS, metoprolol succinate; MT, metoprolol tartarate.

MT cohort. The following characteristics were used to match patients in both cohorts: age, sex, race, ethnicity, income, Charlson comorbidity index (CCI), and insurance type. Based on the balancing guidelines, ${ }^{15}$ the one-to-one caliper matching technique produced the best balance. As a result, patients were matched using the calipers of width equal to 0.2 of the standard deviation of the logit of the propensity score.

To analyze the association between type of metoprolol salt and health care expenditure, a multiple regression analysis using a generalized linear model with a log link function and gamma distribution was used, adjusting for age, sex, race, ethnicity, income, CCI, and insurance type. Total health care expenditure, as defined in MEPS, was the sum of direct payments for care provided during the year, including out-of-pocket payments and payments by private insurance, Medicaid, Medicare, and other sources. The total prescription drug expenditure was the sum of all prescription drugs payments irrespective of the source of payment for the drugs. Interpretation was carried out by taking the natural exponential $[\exp (\beta 1)]$ of the regression coefficient for the expenditure variables. The level of statistical significance was $P \leq 0.05$, and all statistical computations were done with SAS software (v 9.1; SAS Institute Inc, Cary, NC).

\section{Results}

Of the 33,066 unweighted observations, 6658 were identified to use metoprolol. Among these users, 798 were found to be diagnosed for hypertension. After excluding patients who are also diagnosed with angina, the number of patients was reduced to 742. Based on the type of metoprolol they used, 388 patients were found to be using MT and 354 were using MS. After propensity score matching, the two cohorts were further reduced to 291 each to carry out the final analysis. Baseline characteristics (listed in Table 1) were similar in both the cohorts as a result of the propensity score matching.

The average health care expenditure was found to be similar between the two cohorts. The MT cohort had slightly higher average total health care expenditure (US\$10,779). The average prescription drug expenditure was slightly lower in the MT cohort (US\$2675) compared with MS (US\$2761) (Figure 2). The results of the regression analysis showed that the total health care expenditure was not significantly associated with the type of metoprolol product being utilized (Table 2). Upon converting the coefficient into its natural exponential form, the total health care expenditures of MT users was found to be $1.01(P=0.23)$ times higher, than the corresponding expenditures of the MS users; however, 
Table I Demographic characteristics of the MT and MS cohorts

\begin{tabular}{|c|c|c|c|}
\hline & $M T(n=291)$ & MS $(n=29 I)$ & $P$-value \\
\hline \multicolumn{4}{|l|}{ Gender, n (\%) } \\
\hline Male & $138(47.42)$ & I 38 (47.42) & 1.0000 \\
\hline Female & $153(52.58)$ & 153 (52.58) & 1.0000 \\
\hline \multicolumn{4}{|l|}{ Age group, n (\%) } \\
\hline $0-40$ years & $12(4.12)$ & $15(5.15)$ & 0.5544 \\
\hline $4 I-65$ years & $140(48.11)$ & $147(50.5 \mathrm{I})$ & 0.5617 \\
\hline$>65$ years & 139 (47.77) & $129(44.34)$ & 0.4056 \\
\hline \multicolumn{4}{|l|}{ Income, n (\%, USD) } \\
\hline$<20,000$ & $86(29.55)$ & $78(26.80)$ & 0.4610 \\
\hline $20,000-49,999$ & $92(31.61)$ & $97(33.33)$ & 0.6581 \\
\hline $50,000-100,000$ & 80 (27.49) & 80 (27.49) & 1.0000 \\
\hline$>100,000$ & $33(11.35)$ & $36(12.38)$ & 0.7005 \\
\hline \multicolumn{4}{|l|}{ Race, n (\%) } \\
\hline White & $209(71.82)$ & $207(71.13)$ & 0.8543 \\
\hline African-American & $65(22.33)$ & $6 \mathrm{I}(20.96)$ & 0.6873 \\
\hline Other minorities & $17(5.85)$ & $23(7.91)$ & 0.3256 \\
\hline \multicolumn{4}{|l|}{ Ethnicity, n (\%) } \\
\hline Hispanics & $40(13.74)$ & $43(14.77)$ & 0.7221 \\
\hline Non-Hispanics & $25 I(86.26)$ & $248(85.23)$ & 0.7221 \\
\hline \multicolumn{4}{|l|}{ Insurance status, $\mathrm{n}(\%)$} \\
\hline Public insurance & $107(36.77)$ & II 2 (38.48) & 0.6688 \\
\hline Other insurance & $168(57.73)$ & 161 (55.32) & 0.5583 \\
\hline Uninsured & $16(5.50)$ & $18(6.20)$ & 0.7237 \\
\hline \multicolumn{4}{|l|}{ Employment status, n (\%) } \\
\hline Employed & $88(30.24)$ & $96(32.99)$ & 0.4757 \\
\hline Unemployed & $203(69.76)$ & $195(67.01)$ & 0.4757 \\
\hline Charlson's comorbidity index & 2.2 & 2.3 & 0.734 \\
\hline
\end{tabular}

Abbreviations: MS, metoprolol succinate; MT, metoprolol tartarate.

the $P$ value indicated that this association is not statistically significant. In case of prescription drug expenditure, MS users were found to be 1.06 or $6 \%(P=0.019)$ times higher, respectively, than the corresponding expenditures of the MS users.
Among the variables used to adjust for overall health care expenditure employment $(P \geq 0.001)$ status was found to be significantly associated. None of the other variables were significantly associated with the overall health expenditure. In case of prescription drug expenditure, however, along

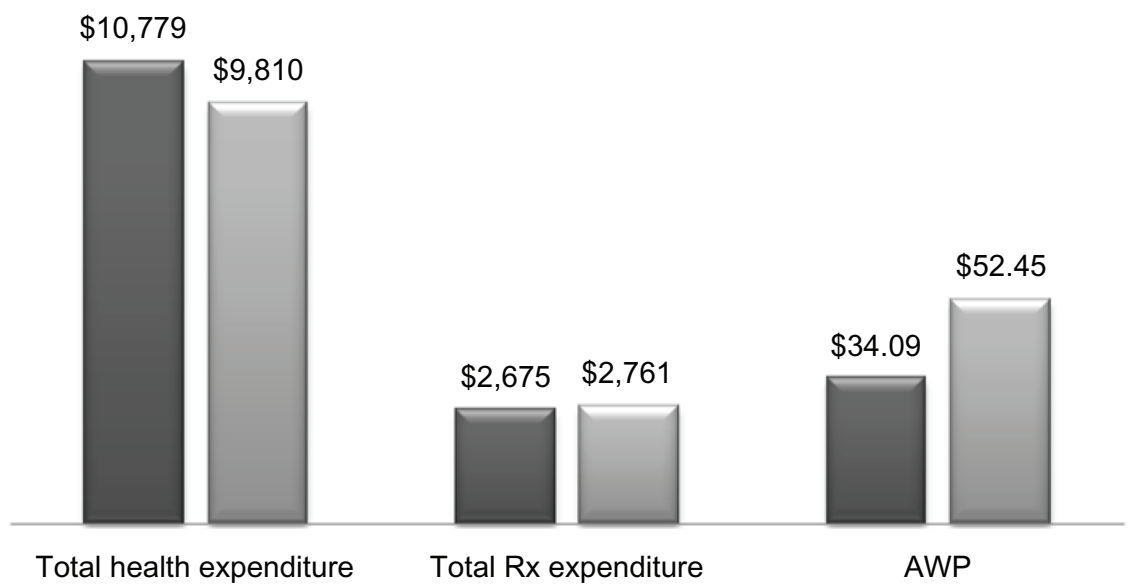

Figure 2 Health expenditure comparison and cost comparison of MT and MS.

Abbreviations: AWP, average wholesale price; MS, metoprolol succinate; MT, metoprolol tartarate; Rx, prescription. 
Table 2 Factors affecting differences in total healthcare and total Rx expenditures

\begin{tabular}{|c|c|c|c|c|}
\hline \multirow[t]{2}{*}{ Variable } & \multicolumn{2}{|c|}{ Total health care expenditure } & \multicolumn{2}{|c|}{ Total $\mathbf{R x}$ expenditure } \\
\hline & Estimate & $P$-value & Estimate & $P$-value \\
\hline Intercept & 2.1153 & $<0.0001$ & 1.8548 & $<0.0001$ \\
\hline \multicolumn{5}{|l|}{ Cohort } \\
\hline MT & Reference & - & Reference & - \\
\hline MS & 0.0148 & 0.2319 & 0.0366 & 0.0208 \\
\hline Comorbidity index & -0.0006 & $0.797 \mid$ & -0.0002 & 0.9526 \\
\hline \multicolumn{5}{|l|}{ Gender } \\
\hline Female & Reference & - & Reference & - \\
\hline Male & -0.0052 & 0.6904 & 0.0101 & 0.5480 \\
\hline \multicolumn{5}{|l|}{ Age (years) } \\
\hline $0-40$ & Reference & - & Reference & - \\
\hline $4 I-65$ & 0.0232 & 0.4461 & 0.0895 & 0.0214 \\
\hline$>65$ & 0.0499 & 0.1201 & 0.1036 & 0.0113 \\
\hline \multicolumn{5}{|l|}{ Income (USD) } \\
\hline$<20,000$ & Reference & - & Reference & - \\
\hline $20,000-49,999$ & -0.0269 & 0.1033 & -0.0183 & 0.3865 \\
\hline $50,000-100,000$ & -0.0016 & 0.9321 & 0.0043 & 0.8574 \\
\hline$>100,000$ & -0.0064 & 0.7930 & -0.0380 & 0.2261 \\
\hline \multicolumn{5}{|l|}{ Race } \\
\hline White & Reference & - & Reference & - \\
\hline African-American & 0.0011 & 0.9482 & -0.0178 & 0.3939 \\
\hline Other minorities & -0.0318 & 0.2064 & -0.0020 & 0.9505 \\
\hline \multicolumn{5}{|l|}{ Ethnicity } \\
\hline Non-Hispanic & Reference & - & Reference & - \\
\hline Hispanic & -0.0141 & 0.4619 & -0.0112 & 0.6484 \\
\hline \multicolumn{5}{|l|}{ Insurance status } \\
\hline Uninsured & Reference & - & Reference & - \\
\hline Public insurance & 0.0185 & 0.5331 & 0.0667 & 0.0776 \\
\hline Private insurance & 0.0253 & 0.3811 & 0.0537 & 0.1462 \\
\hline \multicolumn{5}{|l|}{ Employment status } \\
\hline Unemployed & Reference & - & Reference & - \\
\hline Employed & -0.0940 & $<0.0001$ & -0.0886 & $<0.0001$ \\
\hline
\end{tabular}

Abbreviations: MS, metoprolol succinate; MT, metoprolol tartarate; Rx, prescription.

with employment status ( $P \geq 0.001)$, age categories 40-65 $(P=0.0214)$, and $>65(P=0.0113)$ were also significantly associated.

\section{Discussion}

Previous research on comparing different formulations of the same drug molecule has been mostly limited on establishing efficacy or effectiveness of products. In the case of metoprolol, existing literature documents clinical outcomes associated with MT and MS, ${ }^{12,13}$ but no research is available on how these two products compare with each other in terms of total health care costs incurred. The results of the current study show that the overall expenditures of hypertensive patients on twice-daily MT are not significantly different than the overall expenditures of once-daily MS. However, since the cost of a once a daily formulation is almost two times a twice-daily formulation, MS is associated with significantly higher out-of-pocket cost. The similarities in overall health care expenditure indicate that both the products are equally beneficial, and once daily MS may not offer any additional advantage to justify the additional drug cost.

Adherence is a major issue in the treatment of hypertension, especially if approximately $50 \%$ of the hypertensive population discontinues their antihypertensive medication within the first 6-12 months of initiation of therapy. ${ }^{16} \mathrm{MS}$ being a once-daily formulation has a clear advantage over MT in terms of improving adherence by providing more ease to the patient. Previous studies have shown that simplification of dosing regimens using once-daily medications and/or combination medications does help in improving adherence to the regimen. ${ }^{6,17-22}$ Most of these studies show that there is no significant reduction in the blood pressure associated with increase in adherence to the treatment regimen with a once-daily regimen when compared with a twice-daily regimen in the treatment of hypertension. ${ }^{18-20,22}$ On the other hand, there are studies that show that once-daily medications 
not only improve adherence, but also decrease blood pressure, and are cost-effective as well..$^{21,23}$ As a result, there is lack of established relationship between adherence and lowering of blood pressure. ${ }^{17,24}$ Hence, in this study, the similarity in overall expenditure questions the advantage of MS having a once-daily dosing regimen, as the total health care expenditure is a good predictor of the overall health status of a hypertensive individual. Currently, very little is known about the extent of difference in adherence required to produce any noticeable effect in terms of overall health status and health care expenditure. Further research is warranted to examine adherence rates in similar cohorts and its impact on overall health status. Although both MS and MT have a similar impact on total health care expenditure, one key difference to be noted is, only MS is indicated for the treatment of heart failure by the FDA. ${ }^{10}$ Heart failure is shown to be associated with hypertension. ${ }^{25}$ Therefore, in heart failure patients who are hypertensive, MS is an obvious choice given its FDA-approved indication for heart failure. Moreover, the pharmacokinetic profile of MT is associated with higher utilization of health care services and inferior outcomes when compared with MS in heart failure patients. ${ }^{26}$ In addition to the inferiority of MT, heart failure is associated with a higher risk of hospitalization, which can lead to increased costs; prevention of hospitalization with the use of MS can save money in the long run in this patient population. $^{27}$

MT is available as a discounted generic in many pharmacies. The discounts available on MT have increased access to this medication, even with hypertensive patients who are uninsured. ${ }^{28}$ This access to medications at a deeply discounted price with the same efficacy will ensure that the hypertensive patients do not go without medications due to lack of insurance. The rising cost of prescription drugs presents a significant challenge to individual consumers. Finding affordable treatment options has become a very important need to reduce financial burden on both patients and payers. Future research should be carried out to produce more evidence towards economic comparison of different dosage forms of widely prescribed drugs such as metoprolol using diverse patient populations to generate potential cost savings without compromising health outcomes.

Some clinicians initiate therapy of MT as a once daily dose; however, with the MEPS database, it becomes difficult to identify these patients. The outcomes of this therapy are yet to be ascertained. Nonetheless, if the outcomes of this therapeutic regimen are similar to that of MT given twice a day and/or MS given once a day, there could be considerable reduction in the costs associated with the treatment of hypertension using MT. Further clinical and pharmacoeconomic research of this therapeutic regimen could present clinicians with other cost savings.

Given the sample size and the complexity of the characteristics and the disease of hypertension, the socioeconomic characteristics and their association with health care expenditure needs to be carefully interpreted from this study. Previous research has shown that females have higher overall expenditures, but males are associated with more spending on drugs, physician service, and hospital service. ${ }^{29,30}$ Although the results of this study do not show such association, it demonstrates that the expenditure to age correlation is supported by existing literature. ${ }^{29,30}$ In addition, previous studies have shown conflicting results for the association between expenditure and employment status and, hence, the results of our study should be carefully interpreted. ${ }^{29,30}$ In short, with the limitations of this study, which are listed below, such associations may not be fully confirmed based on a single study.

\section{Limitations}

MEPS, being a self-reported survey, is subject to common limitations such as recall bias and missing information associated with all survey-based retrospective datasets. However, the pharmacy records are verified for accuracy and completeness of the data, which includes the NDC of the drug, the date the prescription is filled and/or refilled, the quantity dispensed, the amount paid by the patient, the amount paid by any third-party payers, and the type of third-party payer during any calendar year.

Another limitation was lack of information on the severity of disease. Although the results were adjusted for the comorbidity index, availability of clinical measures such as the blood pressure of the patients, side effects of the medications, or discontinuation of the medication would have been helpful to assess the safety and efficacy of the regimen in each of the cohorts.

Also, with hypertension being a "silent" disease, many hypertensive patients will not be diagnosed with hypertension, and there would be an underestimation of the problem of hypertension. In addition, with the ICD-9 codes limited to just three digits, we lose the specificity that we could otherwise have with a five-digit ICD-9 code. 
Moreover, the management of hypertension is a complex process, considering the comorbidities associated with it. With the MEPS database, it would be difficult to account for all the dimensions of the pharmacotherapy of hypertension. The use of CCI and propensity score matching would help in balancing the comorbidities in each cohort and hence reduce the chance of bias.

\section{Conclusion}

With the increasing health care costs associated with the treatment of hypertension, it is imperative to analyze the treatment options to avoid unnecessary costs for the health care system. The results of this study contribute towards decision making involved in formulary management and by clinicians treating patients with hypertension. Further analysis such as the present one could help to determine cost-saving options across all classes and combination antihypertensive agents that are available in the market.

\section{Acknowledgment}

The authors thank Dr Vincent Mauro for helping to understand clinical aspects of this study.

\section{Disclosure}

The authors report no conflicts of interest in this work.

\section{References}

1. Cushman WC. The burden of uncontrolled hypertension: morbidity and mortality associated with disease progression. J Clin Hypertens. 2003;5:14-22.

2. MacMahon S, Peto R, Collins R, et al. Blood pressure, stroke, and coronary heart disease. Part 1. Prolonged differences in blood pressure: prospective observational studies corrected for the regression dilution bias. Lancet. 1990;335:765-774.

3. Chobanian AV, Bakris GL, Black HR, et al; for the Committee at NHBPEPC. The seventh report of the joint national committee on prevention, detection, evaluation, and treatment of high blood pressure. JAMA. 2003;289:2560-2571.

4. Rosamond W, Flegal K, Friday G, et al; for the American Heart Association Statistics Committee and Stroke Statistics Subcommittee. Heart disease and stroke statistics - 2007 update. Circulation. 2007;115:e69-e171.

5. Heidenreich PA, Trogdon JG, Khavjou OA, et al. Forecasting the future of cardiovascular disease in the United States. Circulation. 2011;123:933-944.

6. Pittman DG, Tao Z, Chen W, Stettin GD. Antihypertensive medication adherence and subsequent healthcare utilization and costs. Am J Manag Care. 2010;16:568-576.

7. Ormond BA, Spillman BC, Waidmann TA, Caswell KJ, Tereshchenko B. Potential national and state medical care savings from primary disease prevention. Am J Public Health. 2011;101:157-164.

8. Lloyd-Jones DM, Evans JC, Levy D. Hypertension in adults across the age spectrum. JAMA. 2005;294:466-472.

9. Degli Esposti L, Valpiani G. Pharmacoeconomic burden of undertreating hypertension. Pharmacoeconomics. 2004;22:907-928.
10. Dusing R. Optimizing blood pressure control through the use of fixed combinations. Vasc Health Risk Manag. 2010;6:321-325.

11. Efacts. Facts and comparisons [website on the Internet]. Available from: http://www.efactsweb.com/index.asp. Accessed August 15, 2011.

12. Houtzagers JJ, Smilde JG, Creytens G, Westergren G. Efficacy and tolerability of a new controlled-release formulation of metoprolol: a comparison with conventional metoprolol tablets in mild to moderate hypertension. Eur J Clin Pharmacol. 1988;(33 Suppl):S39-S44.

13. Carruthers G, Shearer R, Taylor W, Moore M. A comparison of a controlled release (cr/zok) formulation of metoprolol, once daily, with conventional metoprolol tablets, twice daily, in mild to moderate hypertension. J Clin Pharmacol. 1990;30:S61-S65.

14. Redbook. Montvale, NJ: Thomson Healthcare; 2011.

15. Baser O. Too much ado about propensity score models? Comparing methods of propensity score matching. Value Health. 2006;9: 377-385.

16. Burnier M. Medication adherence and persistence as the cornerstone of effective antihypertensive therapy. Am J Hypertens. 2006;19: 1190-1196.

17. Schroeder K, Fahey T, Ebrahim S. How can we improve adherence to blood pressure-lowering medication in ambulatory care?: Systematic review of randomized controlled trials. Arch Intern Med. 2004;164: $722-732$.

18. Andrejak M, Genes N, Vaur L, Poncelet P, Clerson P, Carre A. Electronic pill-boxes in the evaluation of antihypertensive treatment compliance: Comparison of once daily versus twice daily regimen. $\mathrm{Am}$ J Hypertens. 2000;13:184-190.

19. Baird MG, Bentley-Taylor MM, Carruthers SG, et al. A study of efficacy, tolerance and compliance of once-daily versus twice-daily metoprolol (betaloc) in hypertension. Betaloc compliance Canadian cooperative study group. Clin Invest Med. 1984;7:95-102.

20. Detry JM, Block P, De Backer G, Degaute JP. Patient compliance and therapeutic coverage: Comparison of amlodipine and slow release nifedipine in the treatment of hypertension. The Belgian collaborative study group. Eur J Clin Pharmacol. 1995;47:477-481.

21. Leenen FH, Wilson TW, Bolli P, et al. Patterns of compliance with once versus twice daily antihypertensive drug therapy in primary care: A randomized clinical trial using electronic monitoring. Can J Cardiol. 1997; 13:914-920.

22. Girvin B, McDermott BJ, Johnston GD. A comparison of enalapril $20 \mathrm{mg}$ once daily versus $10 \mathrm{mg}$ twice daily in terms of blood pressure lowering and patient compliance. J Hypertens. 1999;17:1627-1631.

23. Cheng MM, Alfonso R, Best JH, Garrison LP, Bruhn D, Veenstra DL. The economic value of reducing medication dosing frequency with drug delivery technologies: an evidence assessment. J Med Econ. 2010;13:193-202.

24. Schroeder K, Fahey T, Hay AD, Montgomery A, Peters TJ. Relationship between medication adherence and blood pressure in primary care: prospective study. J Hum Hypertens. 2006;20:625-627.

25. Zannad F, Briancon S, Juilliere Y, et al. Incidence, clinical and etiologic features, and outcomes of advanced chronic heart failure: the EPICAL Study. Epidémiologie de l'Insuffisance Cardiaque Avancée en Lorraine. J Am Coll Cardiol. 1999;33:734-742.

26. Tu W, Morris AB, Li J, et al. Association between adherence measurements of metoprolol and health care utilization in older patients with heart failure. Clin Pharmacol Ther. 2005;77:189-201.

27. Braunschweig F, Cowie MR, Auricchio A. What are the costs of heart failure? Europace. 2011;13:ii13-ii17.

28. Rucker NL. $\$ 4$ generics: how low, how broad, and why patient engagement is priceless. JAm Pharm Assoc (2003). 2010;50:761-763.

29. Hodgson TA, Cai L. Medical care expenditures for hypertension, its complications, and its comorbidities. Med Care. 2001;39: 599-615.

30. Hodgson TA, Cohen AJ. Medical care expenditures for selected circulatory diseases: opportunities for reducing national health expenditures. Med Care. 1999;37:994-1012. 


\section{Publish your work in this journal}

ClinicoEconomics \& Outcomes Research is an international, peerreviewed open-access journal focusing on Health Technology Assessment, Pharmacoeconomics and Outcomes Research in the areas of diagnosis, medical devices, and clinical, surgical and pharmacological intervention. The economic impact of health policy and health systems

organization also constitute important areas of coverage. The manuscript management system is completely online and includes a very quick and fair peer-review system, which is all easy to use. Visit http://www.dovepress.com/testimonials.php to read real quotes from published authors.

Submit your manuscript here: http://www.dovepress.com/clinicoeconomics-and-outcomes-research-journal 